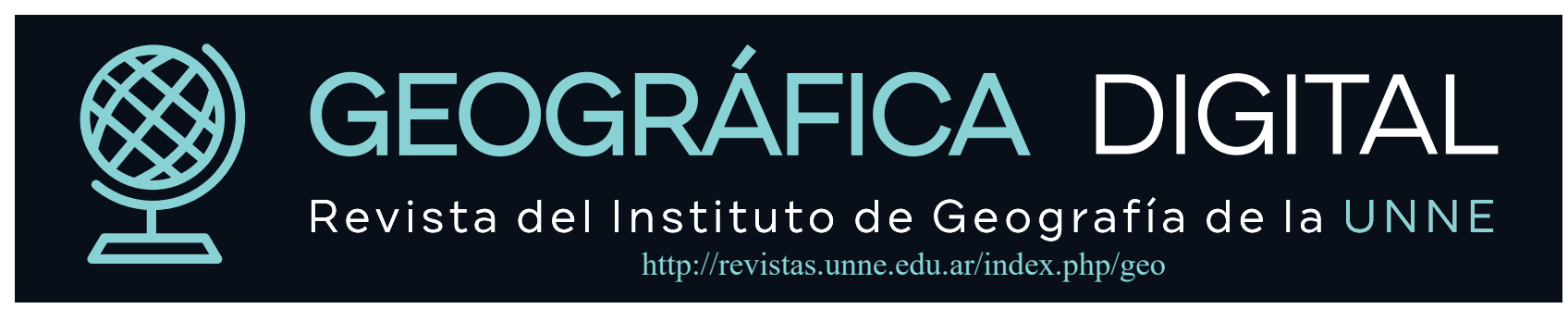

Revista Geográfica Digital, 2021, Vol. 18, No 36, 135-148 pp., E-ISSN: 1668-5180: DOI: http://dx.doi.org/10.30972/geo.18365822

\title{
Análisis de la dinámica de las coberturas de la tierra en el este chaqueño entre 2015 y 2019
}

\section{Analysis of land cover dynamics in eastern of Chaco between 2015 and 2019}

\author{
Molo, Cecilia X. ${ }^{1}$; Cardozo, Osvaldo D. ${ }^{2}$ \\ 1'Instituto de Investigación para el Desarrollo Territorial y Hábitat Humano (IIDTHH) CONICET-UNNE. molo.ximena@gmail.com \\ ${ }^{2}$ Instituto de Investigación para el Desarrollo Territorial y Hábitat Humano (IIDTHH) CONICET-UNNE. Departamento de Geografía. Facultad de \\ Humanidades. Universidad Nacional del Nordeste (UNNE). odcardozo@hum.unne.edu.ar
}

\begin{abstract}
R E S U M E N
Palabras Claves:

Chaco

Cobertura de la tierra

Análisis de cambios

Sistemas de Información Geográfica

Los cambios en el uso y cobertura de la tierra son un proceso dinámico y complejo que vincula la acción recíproca entre los sistemas naturales y antrópicos de un territorio. Para cualquier intento de planificación territorial, es necesario medir espacial y temporalmente las modificaciones. En este sentido, la cartografía de cobertura de la tierra basadas en técnicas de procesamiento de imágenes satelitales, permite generar resultados estadístico-cartográficos que ayudan a comprender mejor los cambios, y su impacto en el ambiente.

Gran parte del este chaqueño es un sitio Ramsar, con una amplia diversidad biológica y de ambientes que le imprimen un carácter único, pero también una gran fragilidad ante cambios que alteren su equilibrio. La cartografía de cambios es un insumo fundamental para gobiernos, instituciones, e investigadores relacionados al cambio climático, la conservación y el aprovechamiento sustentable de los recursos naturales.

El insumo principal de información fueron datos ráster, ofrecidos por el Copernicus Global Land Service con resolución espacial de 100 m en los años 2015 y 2019. Para el procesamiento se empleó un software GIS de propósito general, mientras que el análisis de los cambios se efectuó con un módulo específico denominado Land Change Modeler.

Con el objeto de visualizar la distribución espacial de los procesos de cambios y permanencias, se cartografiaron las transiciones sistemáticas en términos de pérdidas y de ganancias. En este proceso se observa que la mayor ganancia fue para humedal ( $50.000 \mathrm{ha}$ ), mientras que las mayores pérdidas fueron en herbáceas y bosque con 28.000 y 22.000 ha respectivamente.
\end{abstract}

Keywords:

Chaco

Land cover

Change analysis

Geographic Information System

\section{A B S T R A C T}

Changes in land use and coverage are a dynamic and complex process that links reciprocal action between the natural and anthropic systems of the territory. For any attempt at territorial planning, it is necessary to measure the spatially and temporally modifications. In this sense, land cover cartography based on processing techniques satellite image allows the generation of statisticalcartographic results that help to get a better understanding of changes and their impact on the environment.

Much of eastern Chaco is a Ramsar site, with extensive biological diversity, environments with unique character, but on the other side fragile in the face of changes that alter its balance. The mapping of changes is a fundamental input for governments, institutions, and researchers related to climate change, conservation and sustainable use of natural resources. 


\begin{abstract}
The main information input was raster data, offered by the Copernicus Global Land Service with a spatial resolution of $100 \mathrm{~m}$ between 2015 and 2019. General-purpose GIS software was used for the processing, while the analysis of the changes was carried out with a specific module called Land Change Modeler. To visualize the spatial distribution of the processes, change and permanence systematic transitions were mapped in terms of gains and losses. In this process, it is observed that the greatest gain was for wetlands (50,000 ha), while the greatest losses were in herbaceous and forest with 28,000 and 22,000 ha respectively.
\end{abstract}

\title{
1. Introducción
}

Las modificaciones ocurridas en las coberturas de la tierra en un área geográfica determinada, son el resultado de las interacciones entre las actividades antrópicas (asentamientos humanos, actividades productivas) y el medio natural circundante. El cambio en el uso de la tierra es un proceso dinámico y complejo que vincula los sistemas naturales y antrópicos que existen en el territorio. Asimismo, las alteraciones climáticas y las modificaciones en la composición atmosférica, también modelan el paisaje produciendo un cambio en las coberturas de la tierra. Estas modificaciones se presentan en el ámbito local o regional y a escala global (Paruelo et al., 2006). Dichos cambios dependiendo de su magnitud pueden ser más o menos evidentes y observables a través del paisaje, lo cual se ajusta a la escala que percibe el ser humano, con los problemas de subjetividad que ello acarrea.

En este sentido, es necesario medir espacial y temporalmente tales cambios, paso necesario para la planificación del uso de la tierra y la posterior evaluación del impacto y las consecuencias del cambio. Por ello, los datos sobre la cobertura global de la tierra son un recurso clave de información para comprender las complejas interacciones entre las actividades humanas y el cambio global (Running, 2008). Así lo entienden desde la European Space Agency (ESA) al incluir un componente específico llamado Copernicus Global Land Service (CGLS) dentro del programa dedicado a la observación terrestre conocido como Land Monitoring Core Service (LMCS), que ofrece mapas globales de coberturas de la tierra generadas a partir del procesamiento de imágenes satelitales bajo un sistema clasificatorio estándar, permitiendo de esta forma hacer comparaciones para analizar la dinámica de los cambios.

Los mapas de cobertura de la tierra representan información geográfica sobre diferentes tipos de cobertura física observables de la superficie terrestre, por ejemplo bosques, pastizales, cultivos, lagos, humedales, etc., mientras que los mapas de uso de la tierra contienen información sobre las actividades que las personas realizan en un determinado tipo de cubierta terrestre para producirla, cambiarla o mantenerla. Esta primera aproximación ya deja en evidencia, la estrecha relación entre la cobertura de la tierra y los usos de la misma, y es por ello que los cambios de cobertura y uso generalmente se estudian en forma conjunta (Meyer \& Turner, 1994).

Cuando hablamos específicamente de la cobertura de la tierra, hacemos referencia a las características físicas y biofísicas que se observan sobre la superficie terrestre, por lo tanto, en términos amplios, no solo describe la vegetación natural (arbórea, arbustiva, herbácea) y los elementos antrópicos de la actividad humana (cultivos, obras de infraestructura, edificios, canales, etc.) presentes en el territorio, también describen otras superficies como afloramientos rocosos, suelo desnudo, cursos y cuerpos de agua. Por su parte, el término uso hace referencia al propósito o la forma en que se manejan los tipos de coberturas y normalmente tienen una connotación económica, por ejemplo: producción forestal, industrial, ganadera, agrícola, etc., o socio-culturales como recreación, áreas protegidas, hábitat humano, etc. (Meyer \& Turner, 1992; Di Gregorio \& Jansen, 2005).

La importancia de contar con mapas de la cubierta terrestre de calidad, que sean coherentes a lo largo de una serie de años y a escala global, obedece a que proporcionan al mismo tiempo una visión general y sinóptica del estado de las coberturas, así como la posibilidad de detectar sus cambios en el espacio y el tiempo. En el actual contexto de discusión sobre cambio global y cambio climático en los organismos mundiales y prácticamente todos los países, disponer de esta información geográfica resulta crítica.

La información sobre los cambios en la cobertura y/o uso de la tierra es utilizada por administradores de recursos -naturales, humanos, financieros, etc.-, decisores políticos, planificadores y científicos que estudian el ciclo mundial del carbono, pérdida de biodiversidad, degradación del suelo, deforestación, desertificación, expansión de cultivos o de áreas urbanas, entre otras cuestiones vinculadas a presión humana sobre el sistema natural. A partir de ellos se derivan muchas aplicaciones en agricultura y seguridad alimentaria, desarrollo urbano, regional y rural, ordenamiento y planificación territorial, gestión de los recursos hídricos, conservación del capital natural, etc.

Cabe señalar que los sitios con mayor diversidad biológica y de ambientes son también los más 
vulnerables a los procesos de cambio global y cambio climático (Chuvieco, 2008). Uno de ellos corresponde a la región oriental de la provincia del Chaco, un área bajo protección de sitios Ramsar ${ }^{1}$ que busca mantener las características ecológicas y de biodiversidad a través de enfoque por ecosistemas en un contexto de desarrollo sostenible, para lo cual, la información de base referida a las principales coberturas de la tierra son un punto de inicio obligado.

Por esta razón, el objetivo central es analizar la dinámica del cambio en las coberturas de la tierra durante el período 2015-2019 en el sector este de la provincia del Chaco mediante herramientas SIG, tomando como información de base los mapas de cobertura global a $100 \mathrm{~m}$. De manera particular, se han planteado los siguientes objetivos:

- Realizar un análisis multitemporal de la dinámica de cambio en la cobertura de la tierra en el sector oriental de la provincia del Chaco durante el período 2015-2019.

- Elaborar la cartografía de cambios, permanencias e intercambios entre las coberturas de la tierra presentes en el área de interés.

El trabajo se organiza de la siguiente manera. En la primera sección se expone la relevancia del tema, algunos conceptos estructurantes y los objetivos. En la segunda se comentan los antecedentes más relevantes a nivel global y local. En el apartado materiales y métodos, se presenta el área de estudio, las características de la información geográfica utilizada y las técnicas de análisis empleadas para detectar los cambios en las coberturas. En el cuarto se muestran los resultados más significativos derivados del análisis. En quinto lugar se exponen algunas consideraciones finales y por último las referencias bibliográficas consultadas.

\section{Antecedentes}

La cartografía de coberturas de la tierra a escala continental y mundial de forma continua fue posible gracias al acceso abierto de grandes conjuntos de datos espaciales, teledetección con resolución temporal y espacial adecuadas, así como mayores capacidades de procesamiento gracias a la utilización de software en la nube (Gorelick et al., 2017).

Los primeros mapas globales de cobertura de la tierra aparecen en la década de 1990, generados a partir del sensor AVHRR -Advanced Very High Resolution Radiometer-, mediante una clasificación de máxima probabilidad y árboles de decisión a un resolución espacial de 8 km (DeFries \& Townshend, 1994; Hansen, DeFries, Townshend, \& Sohlberg, 2000). También el International Geosphere-Biosphere Programme logró el producto DISCover Maps a $1 \mathrm{~km}$ de resolución espacial por métodos de clasificación no supervisada (Loveland et al., 2000). Por su parte, la Universidad de Maryland generó su mapa global de coberturas de la tierra con píxeles de $1 \mathrm{~km}$ mediante clasificación supervisada (Hansen et al., 2000). A partir del año 2000, se generó el mapa GLC2000 de cobertura global a 1 km mediante imágenes SPOT (Bartholome \& Belward, 2005), así como otro de igual resolución espacial a partir de datos del sensor MODIS utilizando distintos tipos de algoritmos clasificatorios (Tateishi et al., 2011). Recién el mapa de cobertura global GlobCover generado con imágenes MERIS -Medium Resolution Imaging Spectrometer- para los años 2005 y 2009, logró reducir el tamaño del píxel a 300 m (Arino et al., 2008).

La producción de mapas de cobertura de la tierra a gran escala con una mejora sustancial en la resolución espacial, recién fue posible gracias a la utilización de información proveniente de la serie Landsat y Sentinel-2 (Gong et al., 2013; Hansen et al., 2013). En este sentido, cabe mencionar el producto de coberturas de la tierra para África con una resolución espacial de $100 \mathrm{~m}$, experiencia a partir de la cual fue generado el mapa global con idéntico tamaño de píxel por el Copernicus Global Land Service (Buchhorn et al., 2020).

En el contexto Latinoamericano, Cardozo \& Bondar (2019) hacen una revisión del estado de situación de las coberturas y usos de la tierra a nivel de países, prestando especial atención a los distintos sistemas utilizados para clasificar las coberturas, lo cual puso de relieve cierta anarquía en los sistemas empleados, así como la necesidad de mejorar la organización y generación de esta información, para que sea válida la comparación entre países.

Los antecedentes de aplicación para el ámbito chaqueño son escasos, sin embargo, algunos aportes recientes exploraron la temática en distintos sectores de la provincia. Insaurralde (2012) estudió la dinámica de las coberturas en el sudoeste chaqueño entre 1989 y 2000 para medir la relación entre la reducción del monte nativo y el aumento de la superficie dedicada al cultivo. Arias et al. (2019) y Saucedo \& Cardozo (2019), estudiaron la dinámica en las coberturas para el norte provincial, utilizando imágenes 
Landsat y el sistema clasificatorio desarrollado por la UN-FAO; aunque los resultados no son concluyentes, ponen en evidencia los cambios ocurridos entre los 15/20 años tomados de referencia para la cobertura de Bosque hacia otras como Cultivo, Humedal, o Suelo desnudo, lo que denota un cambio real equivalente al $8 \%$ de la superficie considerada. También a partir de categorías de UN-FAO pero enfocado en la dinámica de la cobertura urbana del Área Metropolitana del Gran Resistencia -AMGR-, Cardozo et al. (2021) calculan una tasa de cambio del orden del $19 \%$, aunque en un periodo de casi 30 años, donde el fuerte crecimiento del área urbana se produjo a costa de otras coberturas de tipo natural como Humedales y Vegetación Herbácea, quienes redujeron su superficie en un porcentaje equivalente. Recientemente Cardozo, et al. (2021) también para el sudoeste chaqueño, actualizaron el inventario de las coberturas de la tierra en base al sistema clasificatorio LULC de Anderson-USGS y con una mejora en la resolución espacial a partir de imágenes de la plataforma Sentinel-2.

\section{Materiales y métodos}

\subsection{Delimitación espacial y temporal}

La provincia del Chaco se ubica en el noreste de la República Argentina (ver Figura 1), y con una superficie de $99.633 \mathrm{~km}^{2}$ alberga una población de 1.204 .000 aproximadamente, según las proyecciones del Instituto Nacional de Estadística y Censos (INDEC) para el año 2020.

El área de interés se ubica en el sector oriental de la provincia del Chaco comprendiendo a cinco departamentos: $1^{\circ}$ de Mayo, Bermejo, General Donovan, Libertad, y San Fernando, quienes según el último Censo Nacional de Población, Hogares y Viviendas concentran el 41,5 \% de la población total de la Provincia (437.750 personas) en apenas el 10,5\% de la superficie provincial, situación que se explica por la presencia del principal núcleo urbano: Resistencia y su área metropolitana.

Figura 1. localización del área de estudio en la Provincia del Chaco (izquierda y centro).
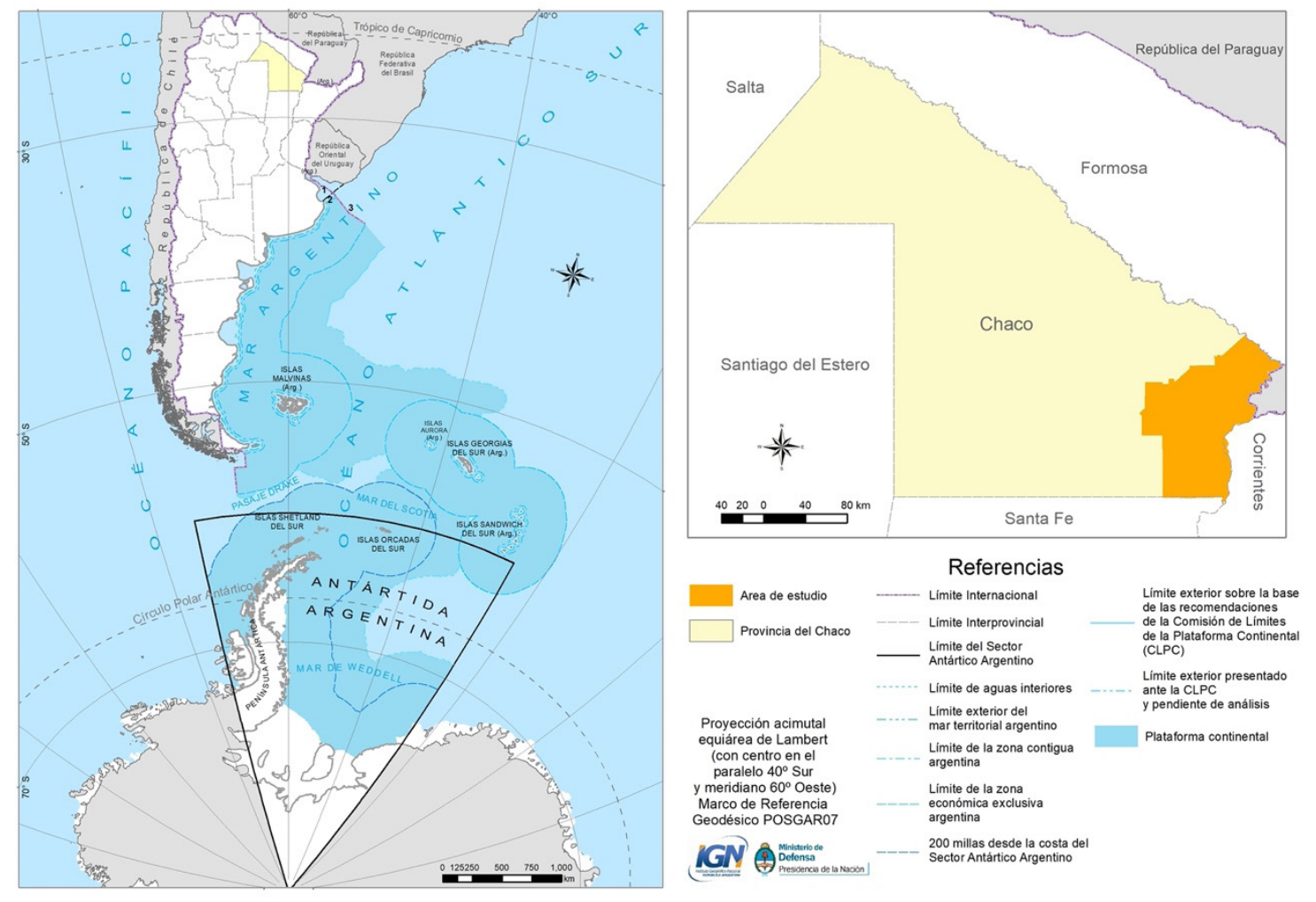

Fuente: IGN-elaboración propia.

Su relieve es una continuidad de la tendencia general en la provincia, una llanura muy plana con leve inclinación en dirección noroeste-sudeste. Esta tendencia se ve reflejada en el sistema de avenamiento y la red hidrográfica, cuyos principales cursos aunque adquieran un carácter meándrico debido a la escasa pendiente, escurren en forma paralela al río Bermejo alternando valles y albardones poco marcados en la 
misma dirección. Este patrón se ve interrumpido en el sector sur -a partir de Resistencia aproximadamente-, donde el río Paraná ensancha su valle principal y el escurrimiento es menos claro, dando lugar a un área sujeto a inundaciones estacionales que combina la presencia de numerosos bañados, esteros y lagunas con el modelo anastomosado del Paraná (Bruniard,1975-78).

La presencia de vegetación es abundante debido a que las precipitaciones de entre 1.000 y 1.200 $\mathrm{mm}$ anuales generan excesos de agua. Se destaca la presencia de vegetación hidrófila en los cuerpos y cursos de agua, mientras que sobre los albardones se desarrolla un monte nativo denso con especies de gran porte. En las planicies se desarrollan herbáceas y arbustivas (Alberto, 2006), y donde la capacidad de los suelos lo permite -sector sur-, se desarrollan prácticas agrícolas de cierta envergadura.

\subsection{Datos}

El recorte temporal obedece a la serie de datos disponibles por parte del consorcio Copernicus entre los años 2015 y 2019. La información disponible en forma de mosaicos de 20x20 grados del servidor de mapas de coberturas (ver Figura 2), es generada y publicada por el equipo del Copernicus Global Land Service (CGLS), un componente del Land Monitoring Core Service (LMCS) de Copernicus, utilizado principalmente para el monitoreo del estado y los cambios en la cubierta terrestre. El primer ensayo de este producto se realizó en el continente africano, y a partir de esa experiencia fue posible extenderlo a todo el mundo (Buchhorn et al., 2020).

Figura 2. Visor web para visualización y descarga de las coberturas de la tierra a nivel mundial.

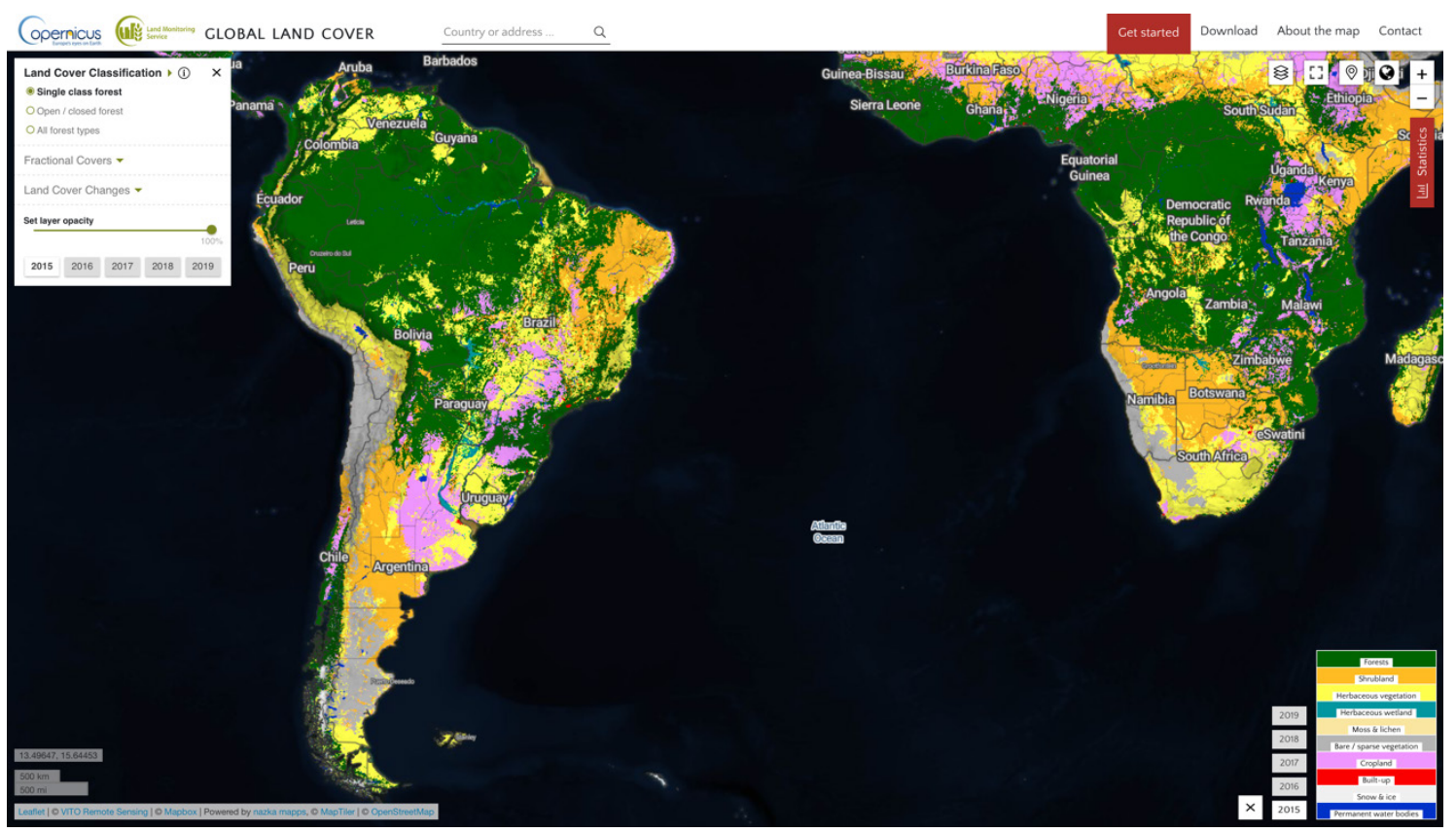

Fuente: https://lcviewer.vito.be/2015

Se trata de un conjunto de mapas a escala global para los años 2015, 2016, 2017, 2018 y 2019, con resolución espacial de $100 \mathrm{~m}$ y una precisión general del 80\%, logrado a partir del empleo de imágenes del satélite PROBA-V, aplicando fusión de observaciones a 100 m y 300 m, junto a métodos de clasificación supervisada random forest. Con el objeto de lograr la precisión del $80 \%$-un valor muy bueno para un producto global-, se emplearon más de 168.000 puntos de entrenamiento del proyecto de colaboración colectiva Geo-wiki, revisados y actualizados por la Wageningen University \& Research de Holanda, junto a 21.000 puntos de validación independientes distribuidos en todos los continentes (Tsendbazar et al., 2021).

Los archivos de coberturas son ofrecidos en formato geoTIF bajo un sistema de coordenadas globales (WGS-84) y proyección geográfica estándar (EPSG:4326), por lo tanto las distancias se expresan en medidas angulares. Por esa razón fue necesario re-proyectarlos al sistema plano oficial en nuestro país (POSGAR-2007) en Faja 5, la más adecuada para la provincia del Chaco. De esta forma, todos los valores de superficie calculados están expresados en hectáreas.

Un problema frecuente al emplear información provenientes de distintas fuentes y productores de datos, es la falta de coincidencia entre categorías del sistema clasificatorio de las coberturas (Cardozo \& Bondar, 2019). Para asegurar la comparabilidad de la información así como su integración con otros 
proyectos, la FAO-UN desarrolló un sistema estándar, el Land Cover Classification System (LCCS) basada en diez (10) clases de coberturas terrestres básicas:

1. Bosque

2. Matorral/arbusto

3. Vegetación herbácea/pasto

4. Musgo y líquenes

5. Suelo desnudo o escasa vegetación

6. Cultivo

7. Construido

8. Nieve y hielo

9. Humedal

10. Agua permanente

Los mapas de coberturas ofrecidos por Copernicus Land Service, emplean el sistema de clasificación principal LCCS de FAO-UN, aunque también proponen seis tipos de bosque cerrado y seis tipos de bosque abierto para un análisis pormenorizado de esta cobertura, además de mar abierto y datos faltantes, dando como resultado un total de 23 clases.

En este trabajo se utilizó el sistema de clases principal, y producto del recorte del área de estudio, se han identificado sólo las categorías que se indican en la Tabla 1, junto al código numérico almacenado en el archivo ráster y el color sugerido para la simbología.

Tabla 1. Descripción de las coberturas (tipo, código, color) del Copernicus Land Service.

\begin{tabular}{|c|c|c|}
\hline Cobertura & Código & Col \\
\hline Bosque & 10 & verde \\
\hline Matorral & 20 & marrón claro \\
\hline Vegetación herbácea & 30 & amarillo \\
\hline Cultivo & 40 & violeta \\
\hline Construido & 50 & rojo \\
\hline Desnudo o escasa vegetación & 60 & gris \\
\hline Agua permanente & 80 & azul \\
\hline Humedal & 90 & celeste \\
\hline
\end{tabular}

El recorte de las coberturas para los años 2015 y 2019 del área de estudio corresponde a la Figura 3, donde se puede observar de manera clara la ubicación del Área Metropolitana del Gran Resistencia, los humedales en todo el sector este, algunos cultivos al sur y hacia el oeste la alternancia de bosque, matorral y vegetación herbácea en los albardones y terrenos menos elevados respectivamente. 
Figura 3. Junto coberturas de la tierra para los años 2015 (derecha, arriba) y 2019 (derecha, abajo).



Fuente: elaboración propia.

\subsection{Técnicas de Análisis}

Los modelos estadísticos son muy utilizados en los análisis del cambio en la cobertura y uso de la tierra, aunque debido a que es necesario el manejo de información geográfica, la tecnología SIG asume un rol cada vez mayor, avanzando hacia el desarrollo de modelos que recojan lo mejor posible la complejidad del tema. En este sentido, la serie técnicas de análisis espacial aplicados a la información geográfica descritas en Giri (2012), resultan fundamentales para medir, evaluar y simular los cambios en las coberturas de la tierra.

Cabe indicar que todas las operaciones de procesamiento normales para la preparación de la información geográfica tales como recorte, reproyección, cálculo de estadísticas y maquetado cartográfico, se realizaron en el software ArcGIS, mientras que el análisis de los cambios en las coberturas se efectuó en el software TerrSet, concretamente a través del módulo Land Change Modeler (LCM) desarrollado por Clark University.

Este módulo integrado en TerrSet (exIDRISI) es un sistema de apoyo a la toma de decisiones para la planificación territorial, relacionado con cambios en las coberturas y usos de la tierra, pérdida de biodiversidad y hábitat, reducción de emisiones, etc. Para ello se enfoca en el análisis y modelado de las relaciones entre variables para explicar estados actuales, predecir impactos y simular futuros escenarios de cambio. Con un flujo de trabajo automatizado e integrado al resto de las herramientas de TerrSet, el modulo LCM está basado en redes neuronales -Multilayer Perceptron- para la fase de entrenamiento, en cadenas de Markov para la estimación del volumen de cambios y en evaluación multiobjetivo para la localización de los cambios y persistencias (Eastman \& Toledano, 2018).

\section{Resultados}

A continuación se muestran los resultados estadísticos y cartográficos más destacados del análisis de cambio en las coberturas, representados por valores de ganancias y pérdidas, cambio neto y las contribuciones al cambio neto por cada cobertura, siempre expresados en hectáreas (ha).

La Figura 4 muestra un balance general de las coberturas entre ambos períodos analizados (2015 y 2019), donde se representa la superficie en ha. correspondiente a las pérdidas (color fucsia) y ganancias 
(color verde) por cada cobertura. Cabe señalar que el signo negativo en la grafica, denota perdida de superficie.

Figura 4. Ganancias y pérdidas (ha) de las coberturas analizadas entre 2015 y 2019.

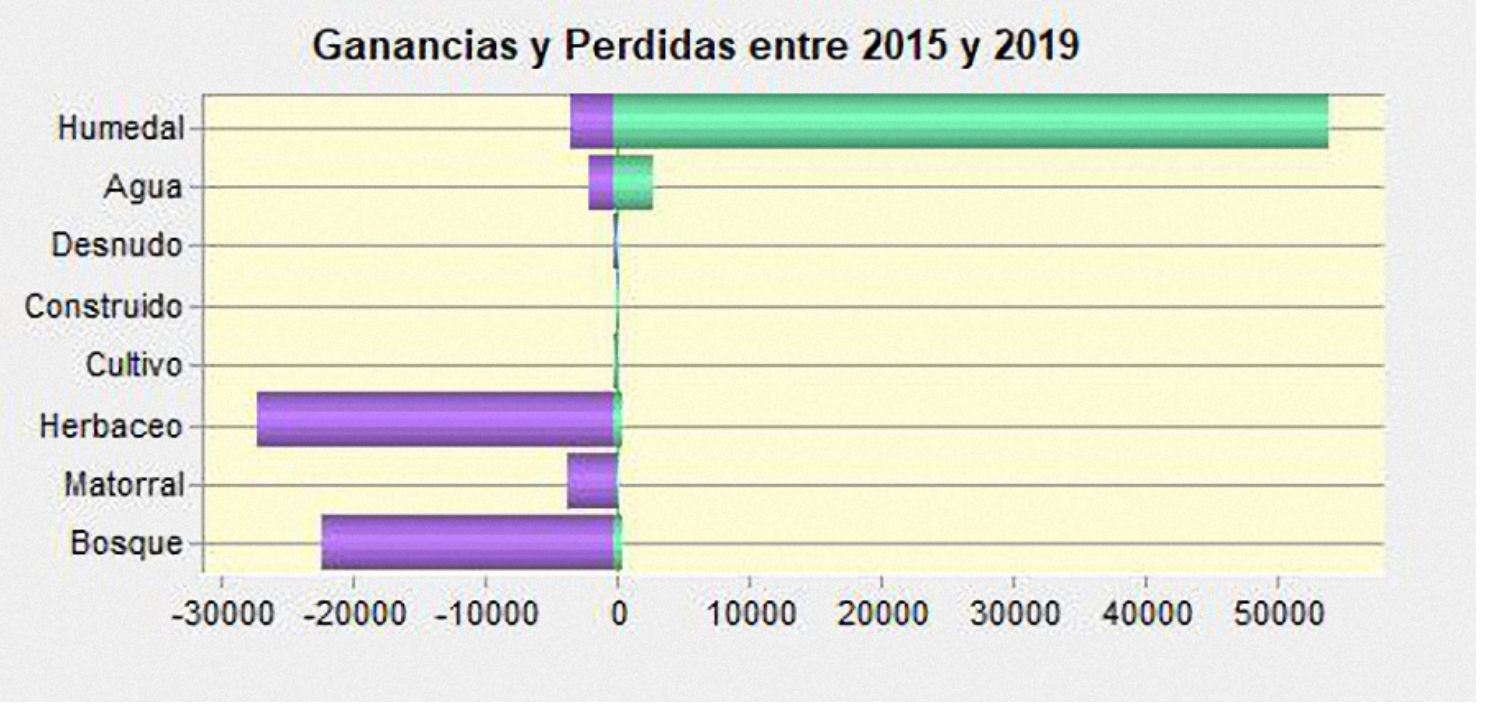

Fuente: elaboración propia.

En principio debemos señalar que de las ocho coberturas analizadas, tres de ellas (Humedal, Herbáceo, Bosque) muestran una dinámica significativa con variaciones entre las 20.000 y 50.000 ha, mientras que dos coberturas (Matorral, Agua) presentan cambios menores (alrededor de $3.000 \mathrm{ha}$ ), y tres de ellas (Suelo desnudo, Construido, Cultivo) se muestran prácticamente sin cambios. Aquí es evidente importante variación positiva que experimenta la cobertura Humedal.

En segundo término, es posible observar las contribuciones individuales a los cambios en cada categoría, siendo de particular interés, aquellas que más cambios han experimentado: Humedal, Herbáceo, y Bosque, aunque también interesan las de Agua y Matorral.

Figura 5. Contribuciones al cambio neto en la cobertura Humedal.



La Figura 5 muestra las tres coberturas de mayor contribución al cambio positivo de la cobertura Humedal: Herbáceo con 25.387 ha, Bosque con 21.592 ha, y Matorral con 3.570 ha. Esto significa que unas 50.000 ha. cambiaron de esas categorías a Humedal entre los años considerados. 
Figura 6. Contribuciones al cambio neto en la cobertura Agua.

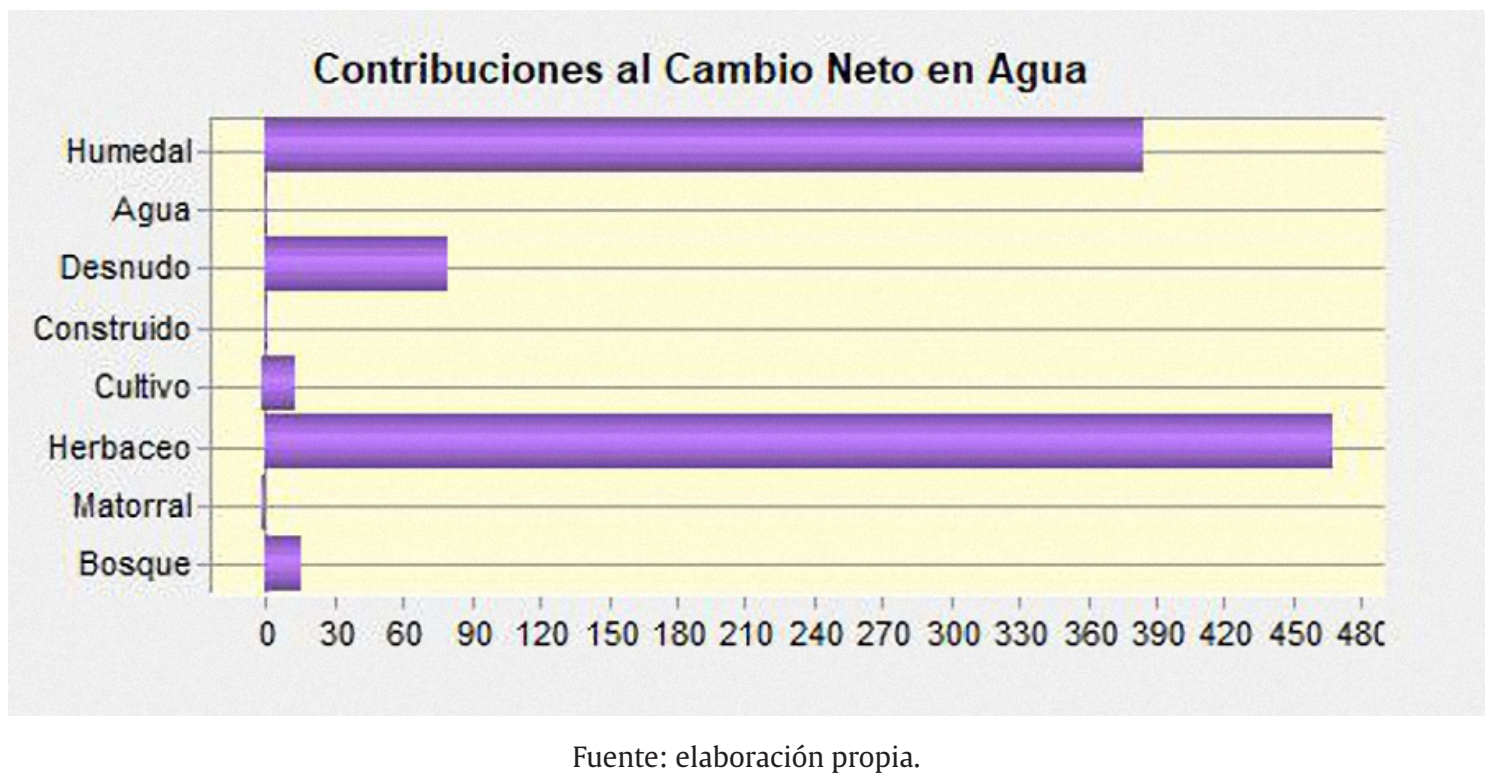

Las contribuciones al cambio neto de la cobertura Agua se observan en la Figura 6. Aunque el volumen de superficie es menor, se destacan los aportes de Humedal y Herbáceo con 400 y 500 ha aproximadamente cada una, seguida por Suelo Desnudo con algo menos de 100 ha, mientras que Bosque y Cultivo aportan menos de 15 ha respectivamente.

Figura 7. Contribuciones al cambio neto en la cobertura Bosque.

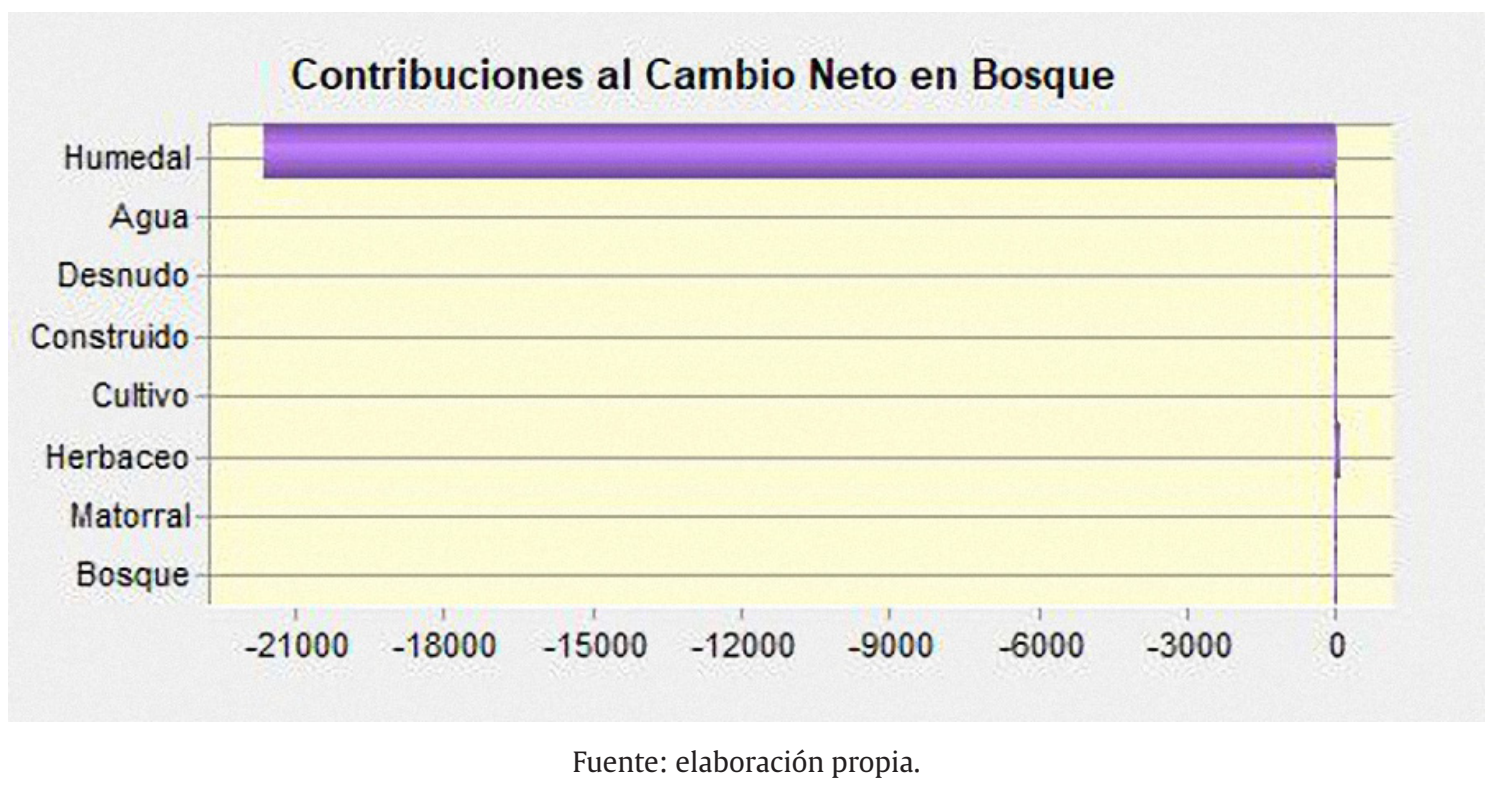

En las Figuras 7 y 8 se pueden observar las contribuciones al cambio neto de Bosque y Matorral respectivamente, siendo notorio que prácticamente todas las pérdidas de superficie -unas 21.000 y 3.500 ha respectivamente-, sean como resultado de sus aportes a la cobertura Humedal. 
Figura 8. Contribuciones al cambio neto en la cobertura Matorral.

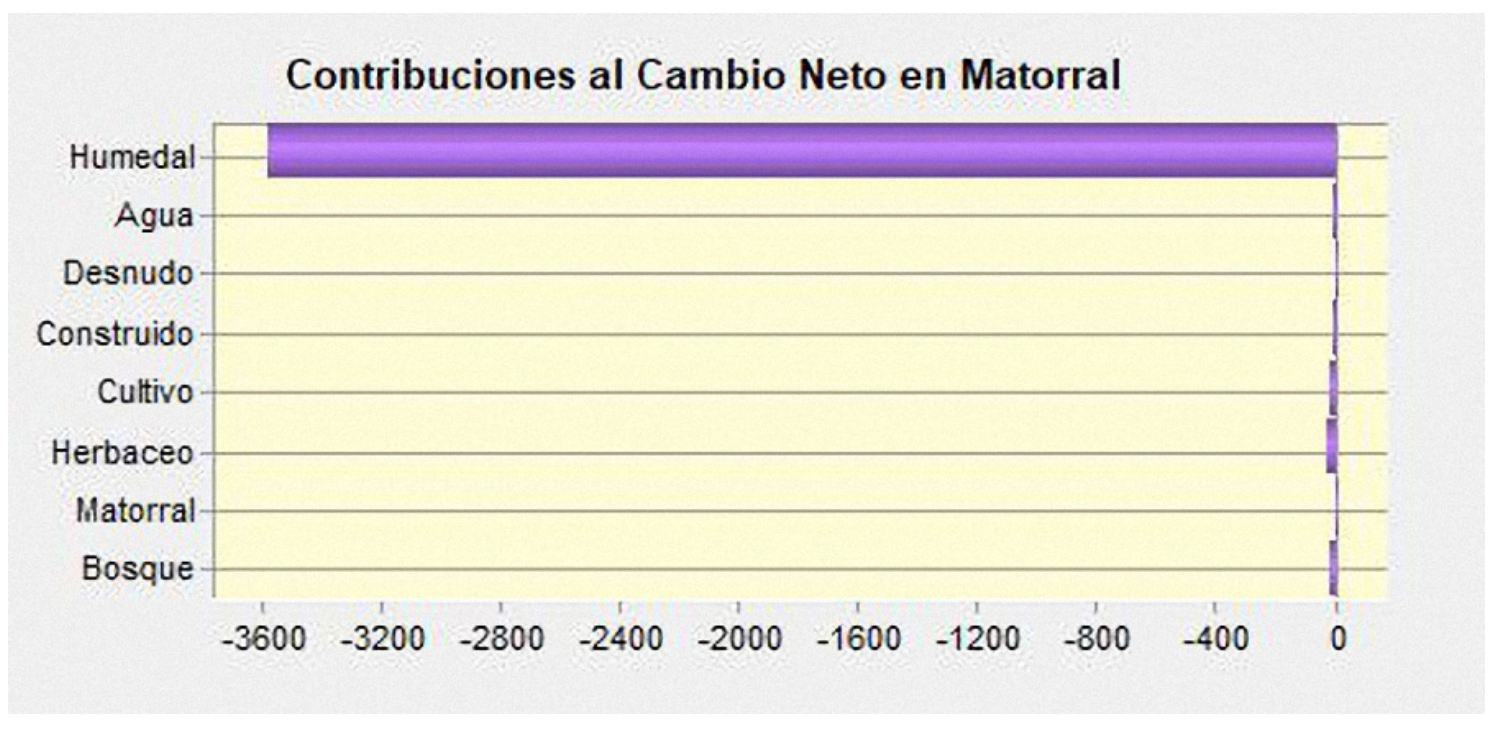

Fuente: elaboración propia.

El cambio neto de la vegetación Herbácea representado en la Figura 9 indica también un comportamiento similar a la grafica de Bosque y Matorral, con un saldo negativo a raíz de la pérdida de unas 24.000 ha que cambiaron a Humedal en el periodo analizado, aunque un residual de superficie muy pequeño fue a parar en Agua y Cultivo.

Figura 9. Contribuciones al cambio neto en la cobertura vegetación Herbácea.



Fuente: elaboración propia.

La cartografía de cambios es muy buena para observar el cambio espacial en las coberturas analizadas. Aunque existen varias alternativas, las más destacadas son el mapa de cambio total donde se comparan todas las categorías, el mapa de persistencias donde figuran las coberturas que no han sufrido cambios, y finalmente el mapa de los intercambios donde se observa el traspaso de pixeles de una cobertura a otra. 
Figura 10. Mapa de cambios (izquierda) y persistencia (derecha) en las coberturas de la tierra entre 2015 y 2019.

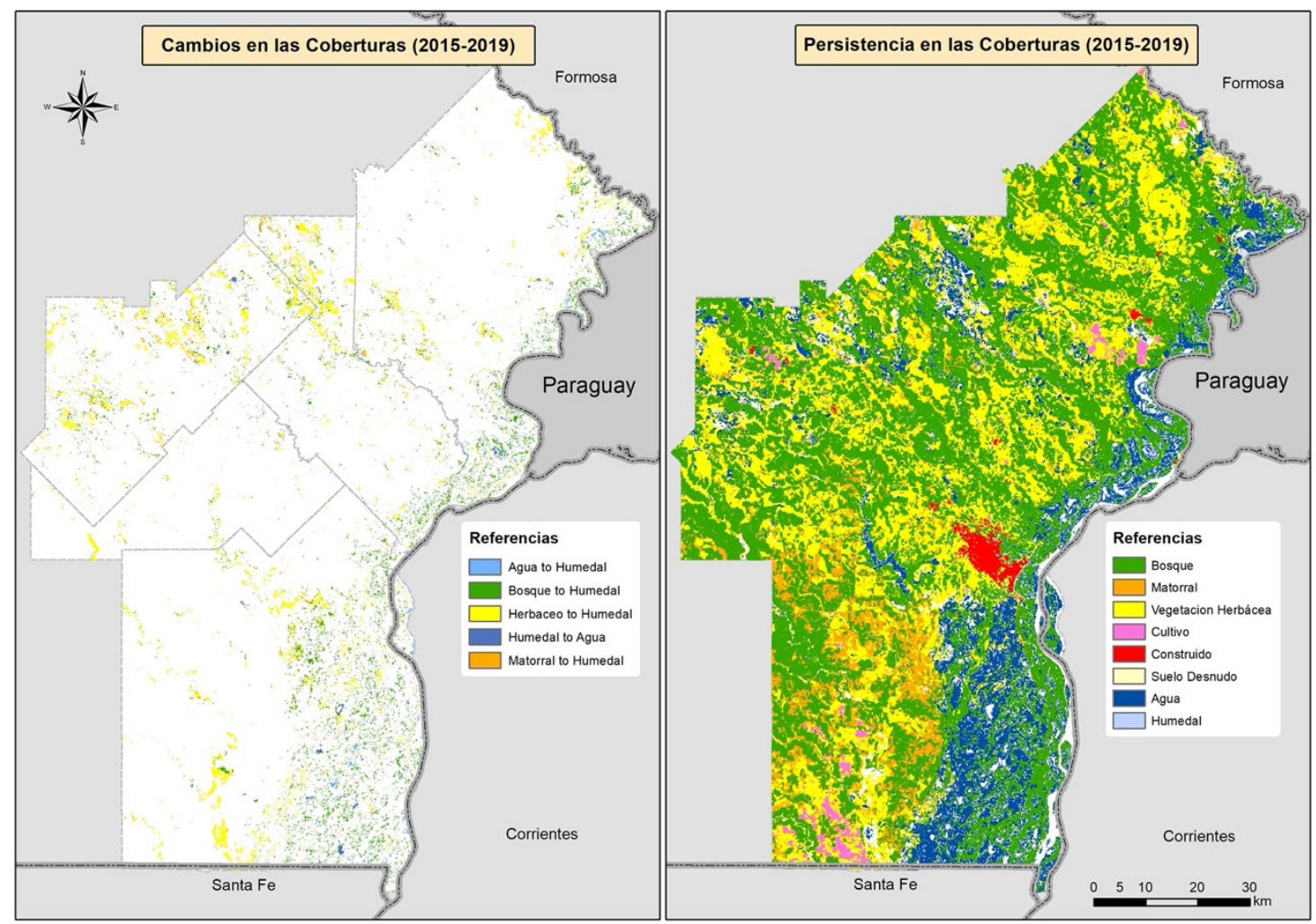

Fuente: elaboración propia.

Los mapas de la Figura 10 representan la persistencia y los cambios en las coberturas de la tierra para el periodo considerado. Como resulta esperable, el mapa de persistencia es similar al de coberturas en 2015, lo cual puede considerarse como bueno, ya que muchos cambios en el territorio no sería una situación deseada. Respecto al mapa de cambios, las categorías que mejor evidencian los cambios producidos -pérdidas particularmente-, son Bosque y Vegetación Herbácea. Aunque muestran patrones de distribución diferentes, son evidentes el Bosque sobre el valle Paraná-Paraguay y Vegetación Herbácea en los sectores más altos al Oeste del área de estudio, sabemos que ambas contribuyeron a la ganancia de superficie para Humedal. 
Figura 11. Mapa de pérdidas y ganancias en las coberturas Bosque (izquierda) y Humedal (derecha) entre 2015 y 2019.

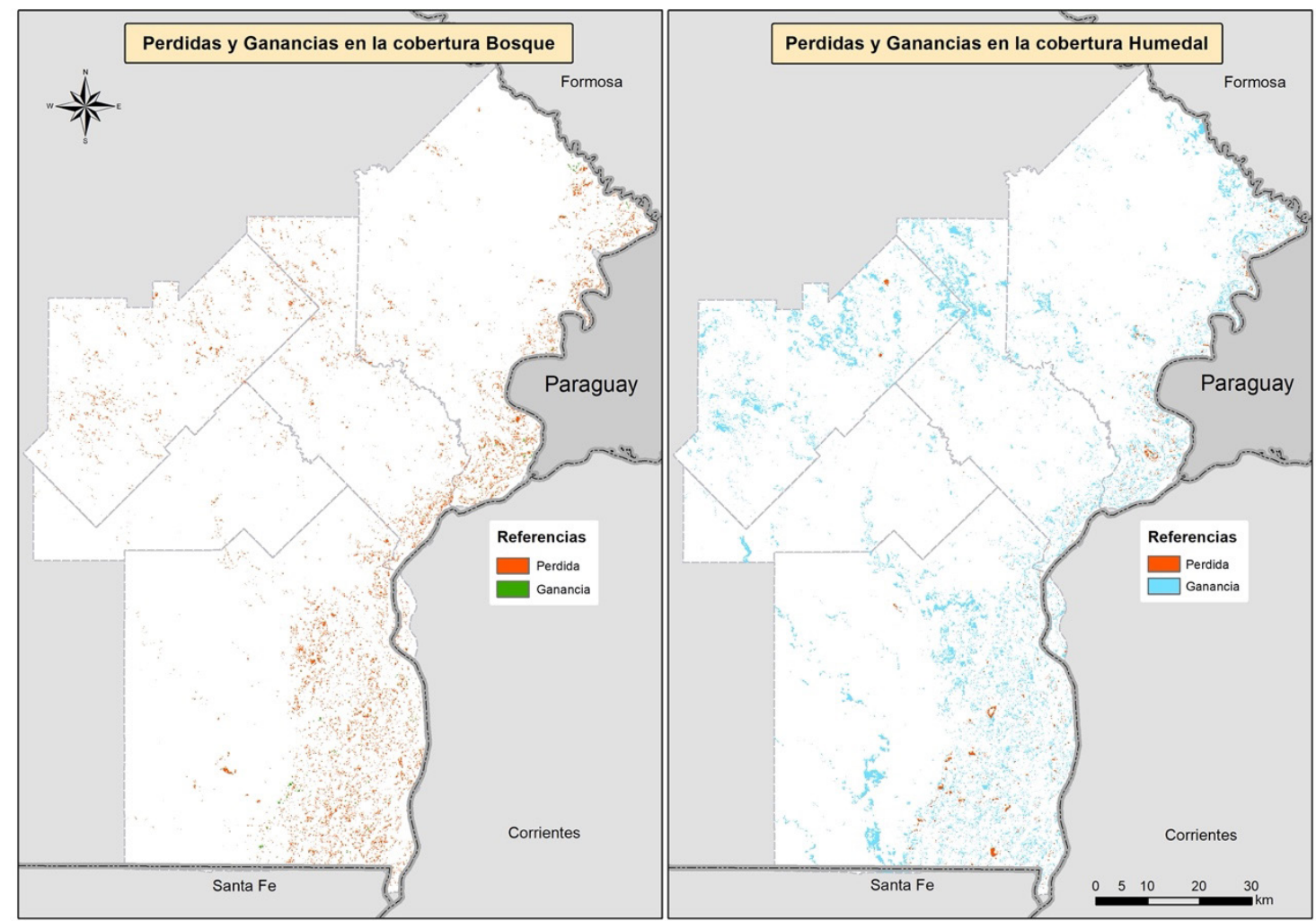

Fuente: elaboración propia.

En la Figura 11 se representa la distribución de las ganancias y pérdidas para las coberturas de Bosque y Humedal. Ambas muestran un patrón de distribución espacial similar que sigue el valle de los ríos Paraguay y Paraná principalmente, seguida por otros sectores hacia el oeste del área de estudio. Esa coincidencia es producto principalmente del cambio de pixeles con categorías de Bosque en 2015 a Humedal en 2019.

\section{Consideraciones finales}

Sin lugar a dudas, la incorporación de los SIG y el Análisis Espacial constituyen un aporte fundamental para el estudio de los cambios en la cobertura y uso de la tierra. Muestra de ello es la creciente aparición de herramientas de software SIG referidos a esta cuestión, así como el número de papers, proyectos, foros, congresos y cursos destinados a la temática.

Con respecto a los resultados del área analizada, es posible afirmar que la dinámica del cambio en las coberturas afectó a unas 118.500 ha, lo cual representa el 11,8 \% de la superficie total considerada. Cabe señalar que al disponer de un periodo de solo 5 años, se trata de un tiempo relativamente corto como para afirmar que todos los cambios en las coberturas sean permanentes.

En cuanto al balance de ganancias y pérdidas, las coberturas Humedal y Agua son quienes han ganado mayor superficie durante el periodo analizado, mientras que las coberturas Herbácea, Matorral y Bosque han resultado con las pérdidas más importantes, al tiempo que en Suelo Desnudo, Construido y Cultivo es casi imperceptible el cambio.

Cabe indicar que si bien el incremento observado en el cambio neto para la superficie ocupada por la cobertura Humedal es innegable, la explicación más lógica es que posiblemente se deba a una variación estacional del agua en superficie, debido a que el año 2019 fue relativamente más húmedo en este sector de la provincia.

Respecto a las contribuciones al mencionado cambio neto, se observa que las cubierta Herbácea, Matorral y Bosque fueron las principales categorías que perdieron superficie en favor de Humedal, mientras que las coberturas Humedal y Herbáceo fueron las responsables del incremento de la superficie ocupada por Agua.

En relación a la serie de datos utilizada, Global Land Cover 100 representa una importante fuente 
de información, estándar, transparente y confiable al momento de generar y procesar la información de coberturas. Cuenta también con buenas perspectivas de continuidad ya que según el Copernicus Global Land Service (CGLS) se trata de una metodología probada, al que se podrían sumar datos de las plataformas Sentinel para extender la serie anual a partir de 2020, y tener la opción de pasar a una mayor resolución espacial, tal como lo promueve el proyecto World Cover 10 de la ESA (https://esa-worldcover.org/en) con un pixel de $10 \mathrm{~m}$.

Por último, cabe señalar que a futuro se pretende hacer foco en los cambios más evidentes detectados en distintos lugares de la provincia, algo que hasta el momento no era posible realizar por la falta de series temporales y a la falta de consistencia en las leyendas de las coberturas clasificadas. Esta situación pone en evidencia la necesidad de recurrir a fuentes globales con categorías generales y resolución espacial media/gruesa, para analizar la dinámica de los cambios. Sin embargo, refuerza la idea de iniciativas que buscan generar datasets locales con varios cortes temporales, pero que guarden relación con un sistema clasificatorio estándar que asegure la comparabilidad con otros trabajos similares.

\section{Agradecimientos}

PI 17H016: Detección y medición de cambios en las coberturas y usos de la tierra en la Provincia del Chaco mediante Tecnologías de Información Geográfica en el periodo 1990 y 2020. SGCyT-UNNE.

\section{Referencias bibliográficas}

Alberto, J.A. (2006). El Chaco Oriental y sus fisonomías vegetales. Geográfica Digital. Año 3, № 5, EneroJunio, ISSN 1668-5180. Instituto de Geografía. Facultad de Humanidades. UNNE. Resistencia, Chaco. doi: 10.30972 /geo.352823

Arias, F.C., Saucedo, G. \& Cardozo, O.D. (2019). Coberturas de la tierra en el departamento Almirante Brown (Chaco). Una comparación entre los años 2000 y 2016. GeoHoy. Año 1, № 1, Mayo-Octubre, ISSN: 2618-5458. Instituto de Geografía. Facultad de Humanidades. UNNE. Resistencia, Argentina. http:// hum.unne.edu.ar/revistas/geohoy/index.htm

Arino, O., Bicheron, P., Achard, F., Latham, J., Witt, R., \& Weber, J.L. (2008). GLOBCOVER the Most Detailed Portrait of Earth. ESA Bulletin. 136, 24-31. European Space Agency.

Bartholomé, E. \& Belward, A.S. (2005). GLC2000: A New Approach to Global Land Cover Mapping from Earth Observation Data. International Journal of Remote Sensing, 26, 1959-1977. doi: $\underline{10.1080 / 01431160412331291297}$

Bruniard, E. (1975-78). El Gran Chaco Argentino. Ensayo de interpretación geográfica. Geográfica, 4. Instituto de Geografía. Facultad de Humanidades. UNNE. Resistencia, Argentina.

Buchhorn, M., Lesiv, M., Tsendbazar, N.E., Herold, M., Bertels, L. \& Smets, B. (2020). Copernicus Global Land Cover Layers - Collection 2. Remote Sensing, 12, 108, 1044. doi: 10.3390/rs12061044

Cardozo O.D., Godoy R.G. \& Insaurralde, J.A (Abril 2021). Las coberturas de la tierra en el sudoeste chaqueño para el año 2020. Congreso Internacional de Ciencia, Tecnología, Innovación y Educación. Universidad Martí. Xalapa, México.

Cardozo, O.D. \& Bondar, C.E. (2019). Estados de situación de coberturas y usos de la tierra en Latinoamérica. En G. Buzai (Comp.), Teoría y Métodos de la Geografía Cuantitativa. Libro 2: por una Geografía Empírica (pp. 185-196). Lujan, Argentina: Universidad Nacional de Lujan.

Cardozo, O.D., Vera, C.A \& Godoy, R.G. (2021). Detección de cambios en las coberturas de la tierra en el Gran Resistencia entre 1990 y 2020. En: GeoHoy. Año 2, № 04, Mayo-Octubre. ISSN 2618-5458. Instituto de Geografía. Facultad de Humanidades. UNNE. Resistencia, Argentina. http://hum.unne.edu.ar/ revistas/geohoy/index.htm

Chuvieco, Emilio.(2008). Earth Observation of Global Change. The Role of Satellite Remote Sensing in Monitoring the Global Environment. Dordrecht, Netherlands: Springer. doi: 10.1007/978-1-4020-6358-9

DeFries, R. \& Townshend, J. (1994). NDVI-derived land cover classifications at a global scale. International Journal of Remote Sensing, 15, 3567-3586.

Di Gregorio, A. \& Jansen, L.J.M. (2005). Sistema de Clasificación de la Cobertura de la Tierra. Conceptos de Clasificación y manual para el usuario. 8va Serie. Roma, Italia: Food and Agriculture Organization of 
the United Nations.

Eastman J.R. \& Toledano J. (2018). A Short Presentation of the Land Change Modeler (LCM). En M.T Camacho Olmedo; M. Paegelow; J-F. Mas; \& F. Escobar (Eds), Geomatic Approaches for Modeling Land Change Scenarios (pp. 481-484). Cham, Switzerland: Springer Nature. DOI: 10.1007/978-3-319-60801-3_36

Giri, C. (2012). Remote Sensing of Land Use and Land Cover. Principles and Applications. Boca Raton, USA: CRC Press. 440 p.

Gong, P., Wang, J., Yu, L., Zhao, Y., Zhao, Y., Liang, L., ... Chen, J. (2013). Finer resolution observation and monitoring of global land cover: first mapping results with Landsat TM and ETM+ data. International Journal of Remote Sensing, 34, 2607-2654. doi: 10.1080/01431161.2012.748992

Gorelick, N., Hancher, M., Dixon, M., Ilyushchenko, S., Thau, D. \& Moore, R. (2017). Google Earth Engine: planetary-scale geospatial analysis for everyone. Remote Sensing Environmental, 202, 18-27. doi: 10.1016/j.rse.2017.06.031

Hansen, M.C., Defries, R.S., Townshend, J.R.G. \& Sohlberg, R.A. (2000). Global Land Cover Classification at $1 \mathrm{~km}$ Spatial Resolution Using a Classification Tree Approach. International Journal of Remote Sensing, 21, (6-7), 1331-1364. doi: 10.1080/014311600210209

Hansen, M.C., Potapov, P.V., Moore, R., Hancher, M., Turubanova, S.A., Tyukavina, A., Thau, D., Stehman, S.V., Goetz, S.J., Loveland, T.R., Kommareddy, A., Egorov, A., Chini, L., Justice, C.O. \& Townshend, J.R.G. (2013). High-resolution global maps of 21st-century forest cover change. Science, 342, 850-853. doi: $10.1126 /$ science. 1244693

Insaurralde, J.A. (2012). La ocupación del suelo en el sudoeste chaqueño entre 1989 y 2010: detección de cambios por medio de la Teledetección (tesis de licenciatura). Departamento de Geografía, Facultad de Humanidades, Universidad Nacional del Nordeste. Resistencia, Argentina. 92 p.

Loveland, T.R., Reed, B.C., Brown, J.F., Ohlen, D.O., Zhu, Z., Yang, L. \& Merchant, J.W. (2000). Development of a Global Land Cover Characteristics Database and IGBP DISCover from $1 \mathrm{~km}$ AVHRR Data. International Journal of Remote Sensing, 21, 1303-30.

Meyer, W.B. \& Turner, B.L. (1992). Human population growth and global land-use/cover change. Annual Review of Ecology and Systematics, 23 (1), 39-61. doi: 10.1146/annurev.es.23.110192.000351

Meyer, W.B. \& Turner, B.L. (1994). Changes in Land Use and Land Cover: A Global Perspective. University Corporation for Atmospheric Research. Office for Interdisciplinary Earth Studies. London, United Kingdom: Cambridge University Press. 549 p.

Paruelo, J.M., Guerschman, J.P., Piñeiro, G., Jobbágy, E.G., Verón, S.R., Baldi, G. \& Baeza, S. (2006). Cambios en el uso de la tierra en Argentina y Uruguay: Marcos conceptuales para su análisis. Agrociencia. Vol. X, N $2,47-61$.

Running, S.W. (2008). Ecosystem Disturbance, Carbon and Climate. Science, 321, 652-653. doi: 10.1126/ science. 1159607

Saucedo, G., \& Cardozo, O.D. (septiembre 2019). Análisis multitemporal de coberturas de la tierra en el Parque Nacional El Impenetrable, Provincia del Chaco (2000 y 2019). 80 Semana Nacional de Geografía. Sociedad Argentina de Estudios Geográficos (GAEA). Corrientes, Argentina.

Tateishi, R. Uriyangqai, B., Al-Bilbisi, H., Ghar, M.A. Tsend-Ayush, J., Kobayashi, T., Kasimu, A., Hoan, N.T., Shalaby, A., Alsaaideh, B., Enkhzaya, T. \& Sato, H.P. (2011). Production of Global Land Cover, GLCNMO. International Journal of Digital Earth, 4, 22-49. doi: 10.1080/17538941003777521

Tsendbazar, N., Herold, M., Li, L., Tarko, A., de Bruin, S., Masiliunas, D., Lesiv, M., Fritz, S., Buchhorn, M., Smets, B., Van De Kerchove, R. \& Duerauer, M. (2021). Towards operational validation of annual global land cover maps. Remote Sensing of Environment, doi: 10.1016/j.rse.2021.112686 